\title{
EXAMPLES OF UNORIENTED AREA-MINIMIZING SURFACES
}

\author{
FRANK MORGAN
}

\begin{abstract}
A comprehensive study is made of constructions of area-minimizing flat chains modulo two. Many have singularities. For instance, any bounded, area-minimizing submanifold of $\mathbf{R}^{n}$ occurs as the singular set of some area-minimizing flat chain modulo two in some $\mathbf{R}^{N}$.
\end{abstract}

Introduction. Given a $(k-1)$-dimensional boundary in $\mathbf{R}^{n}$, one can seek to minimize area in some class of surfaces. We want to take a large class that includes nonorientable as well as orientable surfaces, without restrictions on the singularities or topological type. Such a class appears in geometric measure theory as the flat chains modulo two. The existence and regularity of area-minimizing flat chains modulo two are already well established (cf. [7, 4.2.26; 9]). Unfortunately, examples have been rare.

This paper presents several methods for constructing area-minimizing flat chains modulo two. The first five theorems include the following five examples:

1. a Möbius strip,

2. the graph over a convex region of any function satisfying the minimal surface equation,

3. any minimal disc bounded by a curve $C^{2, \alpha}$ close to a circle,

4. any orientable hypersurface bounded by a connected submanifold of the unit sphere and known a priori to minimize area among oriented surfaces, such as the cone over $\mathbf{S}^{3} \times \mathbf{S}^{3}$ in $\mathbf{R}^{8}$,

5. the union of two area-minimizing flat chains modulo two lying in orthogonal subspaces of $\mathbf{R}^{n}$.

Examples 4 and 5 sometimes contain singularities. Theorems 4 and 5 seem to produce all known examples of area-minimizing flat chains modulo two with singularities. Corollary 5.4 produces the first examples of nonlinear singular sets.

Many of the results of this paper have been known to workers in the field; others are new.

Comparison with the case of oriented surfaces. Examples of surfaces which minimize area in the smaller class of oriented surfaces (integral currents) have been much more abundant. H. Federer [10 or 7, 5.4.19] proves that bounded portions of complex analytic varieties are area-minimizing. R. Harvey and H. B. Lawson [15]

Received by the editors April 6, 1983.

1980 Mathematics Subject Classification. Primary 49F22, 53C42.

Key words and phrases. Area-minimizing, flat chain modulo two, singularity.

(1984 American Mathematical Society $0002-9947 / 84 \$ 1.00+\$ .25$ per page 
give other rich "calibrated geometries" of area-minimizing surfaces. Lawson [18] obtains area-minimizing surfaces invariant under a suitable group of isometries from geodesics in a quotient space. (See also [19].)

Unfortunately these methods seem to break down in the class of flat chains modulo two. In one case [22] symmetry arguments and regularity theory are used to prove the existence of a continuum of area-minimizing flat chains modulo two with a certain boundary, but the proof is not constructive.

Organization of this paper. The statement of each of the six theorems is usually followed by remarks, lemmas and finally the proof.

This work was partially supported by a grant from the National Science Foundation. It was completed while the author was a visiting assistant professor at Rice University.

Preliminaries. H. Federer's treatise [7] serves as a reference for basic definitions, notations and results.

0.1 . Oriented surfaces. For the basic class of $k$-dimensional oriented surfaces in $\mathbf{R}^{n}$, we take the rectifiable currents $\mathscr{R}_{k} \mathbf{R}^{n}$ of geometric measure theory. These surfaces can be built from rectifiable sets. There is no restriction on the singularities or topological type. We also use the subspace $\mathbf{I}_{k} \mathbf{R}^{n}$ of integral currents and the larger space $\mathscr{F}_{k} \mathbf{R}^{n}$ of integral flat chains:

$$
\mathbf{I}_{k} \mathbf{R}^{n} \subset \mathscr{R}_{k} \mathbf{R}^{n} \subset \mathscr{F}_{k} \mathbf{R}^{n} .
$$

One can associate to any $S \in \mathscr{F}_{k} \mathbf{R}^{n}$ a boundary $\partial S \in \mathscr{F}_{k-1} \mathbf{R}^{n}$, a mass or area $\mathbf{M}(S) \in[0, \infty]$, a flat norm $\mathscr{F}(S) \in[0, \infty)$, a support spt $S \subset \mathbf{R}^{n}$, and a positive measure $\|S\|$ on $\mathbf{R}^{n}$. A rectifiable current $R$ has an oriented tangent plane $\vec{R}$ at $\|R\|$-almost all points in $\mathbf{R}^{n}$.

The integral currents are just the rectifiable currents whose boundaries are rectifiable currents. The integral flat chains are sums of boundaries of rectifiable currents and rectifiable currents. It turns out that the rectifiable currents are precisely the integral flat chains of finite mass. The usual definitions in Federer refer to a compact set $K$ which will be inconsequential for us, since we will always be able to assume all our currents have supports inside some large closed ball $K_{0}$.

Any compact oriented $C^{1}$ manifold with boundary or any graph over a compact domain of a Lipschitz function may be viewed as a rectifiable current.

0.2 . Unoriented surfaces. For our classes of $k$-dimensional unoriented surfaces in $\mathbf{R}^{n}$ we take the spaces of flat chains modulo two,

$$
\mathbf{I}_{k}^{2} \mathbf{R}^{n} \subset \mathscr{R}_{k}^{2} \mathbf{R}^{n} \subset \mathscr{F}_{k}^{2} \mathbf{R}^{n},
$$

consisting of congruence classes of integral flat chains. For example, two rectifiable currents $S, T \in \mathscr{R}_{k} \mathbf{R}^{n}$ are congruent modulo two if $S-T=2 R$ for some $R \in \mathscr{R}_{k} \mathbf{R}^{n}$. In particular, reversing the orientation of a rectifiable current does not change its congruence class. The congruence class of $T \in \mathscr{F}_{k} \mathbf{R}^{n}$ is denoted by $T^{2} \in \mathscr{F}_{k}^{2} \mathbf{R}^{n}$.

One can associate to any $S \in \mathscr{F}_{k}^{2} \mathbf{R}^{n}$ a boundary $\partial S \in \mathscr{F}_{k-1}^{2} \mathbf{R}^{n}$, a mass $\mathbf{M}^{2}(S) \in$ $[0, \infty]$, a flat norm $\mathscr{F}^{2}(S) \in[0, \infty)$, a support $\operatorname{spt}^{2} S \subset \mathbf{R}^{n}$, and, if $S \in \mathscr{R}_{k}^{2} \mathbf{R}^{n}$, a 
positive measure $\|S\|^{2}$. It turns out that $\mathscr{R}_{k}^{2} \mathbf{R}^{n}$ consists precisely of the flat chains modulo two of finite mass. An $S \in \mathscr{R}_{k}^{2} \mathbf{R}^{n}$ has an unoriented tangent plane $\vec{S}$ at $\|S\|^{2}$-almost all points in $\mathbf{R}^{n}$. Every $S \in \mathscr{R}_{k}^{2} \mathbf{R}^{n}$ has a representative modulo two $T$; that is, a $T \in \mathscr{R}_{k} \mathbf{R}^{n}$ of multiplicity one such that $S=T^{2} ; \mathbf{M}^{2}(S)=\mathbf{M}(T)$.

Any compact $C^{1}$ manifold with boundary, orientable or nonorientable, or any graph over a compact domain of a Lipschitz function, may be viewed as a flat chain modulo two.

Compactness. The following compactness theorem guarantees the existence of solutions to many variational problems. For any $C>0$ and closed ball $K_{0}$, the set of all flat chains modulo two $S$ supported in $K_{0}$ with

$$
\mathbf{M}^{2}(S) \leqslant C, \quad \mathbf{M}^{2}(\partial S) \leqslant C
$$

is compact under the flat norm. The analogous theorem holds for integral flat chains.

The theory of flat chains modulo two was first developed by W. Ziemer [26] (in 1962) and by W. Fleming [12] (in 1966).

\section{3 . Area-minimizing surfaces.}

Definition. A rectifiable current $S \in \mathscr{R}_{k} \mathbf{R}^{n}$ is called area-minimizing if for all $T \in \mathscr{R}_{k} \mathbf{R}^{n}$ with $\partial T=\partial S, \mathbf{M}(T) \geqslant \mathbf{M}(S)$. Similarly, a flat chain modulo two $S \in$ $\mathscr{R}_{k}^{2} \mathbf{R}^{n}$ is area-minimizing if for all $T \in \mathscr{R}_{k}^{2} \mathbf{R}^{n}$ with $\partial T=\partial S, \mathbf{M}^{2}(T) \geqslant \mathbf{M}^{2}(S)$.

Existence. Given any boundary $B \in \mathscr{F}_{k-1} \mathbf{R}^{n}$ (resp. $\mathscr{F}_{k-1}^{2} \mathbf{R}^{n}$ ) with $\partial B=0$, there is an area-minimizing $S \in \mathscr{R}_{k} \mathbf{R}^{n}$ (resp. $\mathscr{R}_{k}^{2} \mathbf{R}^{n}$ ) with $\partial S=B$. (Such existence follows from compactness.)

Regularity. If $S \in \mathscr{R}_{k} \mathbf{R}^{n}$ and $T \in \mathscr{R}_{k}^{2} \mathbf{R}^{n}$ are area-minimizing, then spt $S-\operatorname{spt} \partial S$ and $\mathrm{spt}^{2} T-\mathrm{spt}^{2} \partial T$ are smooth manifolds except for a singular set of Hausdorff dimension at most $k-2[\mathbf{9}$, Theorem $2 ; 4]$.

The theorems. F. J. Almgren and B. Solomon both have showed me a proof of the following theorem by methods to appear in [3].

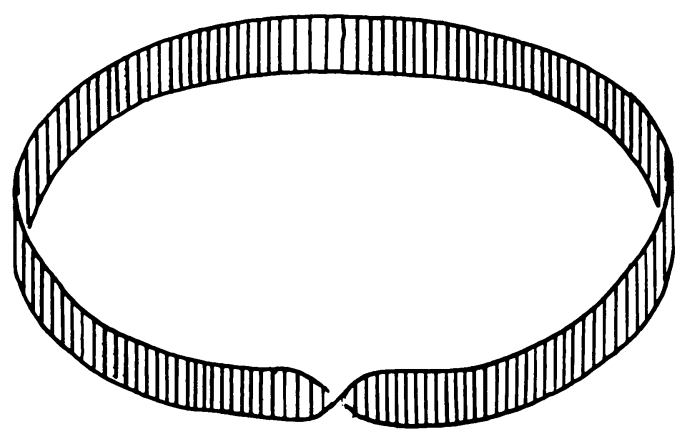

FigURE 1. A Möbius strip 
1. THEOREM. Let $B$ be the smooth curve (which winds twice around a thin torus) parametrized by

$$
\begin{gathered}
f:[0,2 \pi) \rightarrow \mathbf{R}^{3}, \\
f(\theta)=(\cos 2 \theta(1+\varepsilon \cos \theta), \sin 2 \theta(1+\varepsilon \cos \theta), \varepsilon \sin \theta) .
\end{gathered}
$$

For $\varepsilon$ sufficiently small, any area-minimizing flat chain modulo two bounded by $B$ is a Möbius strip, which is an embedded manifold with boundary.

REMARK. The strip can be shown to be close to the strip one would expect.

Remark. Assuming an appropriate bridge theorem, one now deduces the existence of area-minimizing flat chains modulo two of every finite topological type.

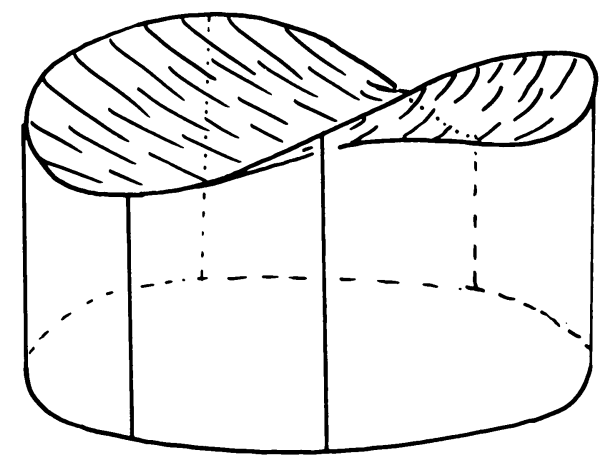

FIGURE 2. The graph of a function satisfying the minimal surface equation

2. Theorem. Let $K$ be a compact, convex domain in $\mathbf{R}^{n}$ and let $f: K \rightarrow \mathbf{R}$ be a Lipschitz weak solution to the minimal surface equation. Then the graph of $f$ is area-minimizing as a flat chain modulo two.

Remark. It follows from [6] that $f$ is real-analytic on the interior of $K$. The theorem is an easy consequence of a maximum principle of R. Hardt [14, 4.1 and 5].

Definitions For THEOREM 3. For any positive integer $q$ and $0 \leqslant \alpha \leqslant 1$, we use $C^{q . \alpha}$ to indicate $\alpha$-Hölder-continuous derivatives of order $q$. For $M$ a compact, $(k+1)$-dimensional, $C^{q, \alpha}$ submanifold with boundary of $\mathbf{R}^{n}, f \in C^{q, \alpha}\left(M, \mathbf{R}^{N}\right)$, let

$$
\begin{array}{r}
\|f\|_{C^{4, \alpha}}=\sup \left\{\left\|D^{j} f(x)\right\|,|x-y|^{-\alpha}\left\|D^{q} f(x)-D^{q} f(y)\right\|:\right. \\
x, y \in M, x \neq y, 0 \leqslant j \leqslant q\} .
\end{array}
$$

Let $p$ denote a fixed real-analytic map from $M$ into the space of $(n-k-1)$ dimensional linear subspaces of $\mathbf{R}^{n}$ such that at each $x \in M, p(x)$ complements the tangent space to $M$ at $x$. Let

$$
C^{q . \alpha}(M, p)=\left\{f \in C^{q . \alpha}\left(M, \mathbf{R}^{n}\right): f(x) \in p(x) \text { for all } x \in M\right\} .
$$

Let $\iota_{M}$ denote the inclusion of $M$ in $\mathbf{R}^{n}$. 


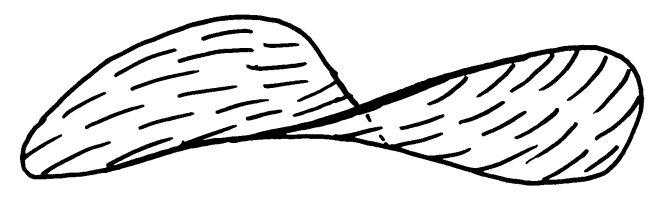

FIGURE 3. An embedded area-minimizing manifold without Jacobi fields

3. THEOREM. For positive integers $n$ and $k, 0<\alpha<1$, let $B$ be a compact, embedded, $k$-dimensional, $C^{q, \alpha}$ submanifold of $\mathbf{R}^{n}$. Suppose that $B$ bounds a unique area-minimizing flat chain modulo two $S$, that $M=\operatorname{spt}^{2} S$ is an embedded manifold with boundary, and that $M$ has no nontangential Jacobi fields which vanish at the boundary. Then given $\varepsilon>0$, there exists $\delta>0$, such that if $\left\|\gamma-\iota_{B}\right\|_{C^{2, \alpha}}<\delta$ then $\gamma_{\#} B$ bounds a unique area-minimizing flat chain modulo two $T, \operatorname{spt}^{2} T$ is an embedded manifold with boundary, and $\gamma$ has an extension $f$ on $M$ such that

$$
T=f_{\#} S \text { and }\left\|f-\iota_{M}\right\|_{C^{2 . \alpha}}<\varepsilon .
$$

3.1. REMARK. The above theorem, an outcome of a conversation with $B$. White, is a consequence of a theorem of his. White's theorem [24, 3.1], under the same hypotheses, concludes that $\gamma_{\#} B$ bounds an embedded minimal manifold $M^{\prime}$, unique among embedded minimal manifolds $C^{2, \alpha}$ close to $M$. Our theorem says that $M^{\prime}$ uniquely minimizes mass among flat chains modulo two with the same boundary.

3.2. Lemma. Let $q, \alpha, M, p, \iota=\iota_{M}$ be as in the introduction to Theorem 3. Given $x \in M, 0<\delta_{1}<\delta_{2}$, there exist $\varepsilon, C>0$ such that if $f \in C^{q . \alpha}\left(M, \mathbf{R}^{n}\right)$,

$$
f \mid \partial M=\mathrm{id}, \quad\|f-\iota\|_{C^{1}}<\varepsilon,
$$

then there is a unique $g \in C^{2, \alpha}(M, p)$ such that $(\iota+g)(M)=f(M)$. Furthermore,

$$
\left\|g\left|\mathbf{U}\left(x, \delta_{1}\right)\left\|_{C^{q, \alpha}} \leqslant C\right\|(f-\iota)\right| \mathbf{U}\left(x, \delta_{2}\right)\right\|_{C^{q, \alpha} .}
$$

REMARK. This lemma can be proved by changing coordinates locally to make $M$ an axis $(k+1)$-plane and $p(x)$ the orthogonal complement to the tangent space to $M$ at $x$.

Sketch of Proof of Theorem 3. Take a sequence of $f$ 's such that

$$
\left\|f-\iota_{B}\right\|_{C^{2, \alpha}} \rightarrow 0 \text {. }
$$

Let $T$ be any area-minimizing flat chain modulo two bounded by $f_{\#} B$. Since $S$ is the unique area-minimizing flat chain modulo two bounded by $B$, one can infer that $T \rightarrow S$ in the flat norm by applying a compactness argument. In fact, the measures $\|T\|^{2} \rightarrow\|S\|^{2}$ (cf. [7, 5.4.2]). Now by covering $M=\mathrm{spt}^{2} S$ by small balls and applying Allard's regularity theorems $[1, \S 8 ; 2, \S 4]$, one concludes that eventually $\mathrm{spt}^{2} T$ is a $C^{1, \alpha}$ manifold with boundary, $C^{1}$ close to $M$.

To state that conclusion more precisely, we adopt the notation of White's paper $[24,3]$. Let

$$
\phi: C^{2, \alpha}\left(B, \mathbf{R}^{n}\right) \rightarrow C^{2, \alpha}\left(M, \mathbf{R}^{n}\right)
$$


be the bounded linear operator such that for $\gamma \in C^{2, \alpha}\left(B, \mathbf{R}^{n}\right), \phi(\gamma)-\iota_{M}$ is the unique harmonic map on $M$ with boundary values $\gamma-\iota_{B}$. It is a standard result that $\left\|\phi(\gamma)-\iota_{M}\right\|_{C^{2, \alpha}} \rightarrow 0$. What follows from Allard's theorem and Lemma 3.2 is that $\operatorname{spt}^{2} T$ can be parametrized as $\phi(\gamma)+g$ for some $g \in C_{0}^{2, \alpha}(M, p)$ with

(1) $\|g\|_{C^{1}} \rightarrow 0,\|g\|_{C^{1 . \alpha}} \leqslant K_{0}$.

We claim that $\|g\|_{C^{2, \alpha}} \rightarrow 0$. Let $\{C\}$ be a finite collection such that:

(2) $C=D \times E$ is the orthogonal product of a small $(k+1)$-ball $D$ and a small $(n-k-1)$-ball $E$ both centered at the same point $a \in M$,

(3) $D$ is tangent to $M$ at $a$,

(4) $C \cap B=\varnothing$ unless $a \in B$,

(5) $C \cap M$ is the graph over $\Omega \subset D$ of a function with gradient less than say .1 in absolute value,

(6) if for $i=1,2,3,4, C_{i}$ denotes $C$ shrunken by a factor $2^{-i}$ by a homothety about $a$, then $\left\{C_{4}\right\}$ covers $M$.

Let $\Omega_{i}=\Omega \cap C_{i}$. We can assume that $(\iota+g)(M) \cap C$ is the graph of a function $w: \Omega \rightarrow E$ with $|\vec{\nabla} w|<.2$, that $\phi(\gamma)(M)$ and $(\phi(\gamma)+g)(M)$ intersect $C$ in graphs over domains in $D$ of functions with gradients less than .2 in absolute value, and that $\phi\left(M \cap C_{1}\right) \subset C$. Define functions $\phi_{1}, \phi_{2}$ on $\Omega$ such that

$$
\begin{gathered}
\phi(\gamma)-\imath: M \cap C_{1} \rightarrow C=D \times E, \\
\phi(\gamma)-\iota=\left(\phi_{1}, \phi_{2}\right) \circ(\text { projection onto } D) .
\end{gathered}
$$

Then $\left\|\phi_{2}\right\|_{C^{2, \alpha}} \rightarrow 0,\left\|\phi_{1}\right\|_{C^{2, a}} \rightarrow 0,\left\|\phi_{1}^{-1}\right\|_{C^{2, \alpha}} \rightarrow 0$. Now since $\operatorname{spt}^{2} T=(\phi(\gamma)+g)(M)$ is minimal, on $\phi_{1} \Omega_{2} \subset D,\left(\phi_{2}+w\right) \circ \phi_{1}^{-1}$ satisfies the minimal surface system [23, Theorem 2.2]

$$
\sum_{i, j=1}^{k+1} G^{i j} \frac{\partial^{2}}{\partial x_{i} \partial x_{j}}\left(w \circ \phi_{1}^{-1}\right)+\sum_{i, j=1}^{k+1} G^{i j} \frac{\partial^{2}}{\partial x_{i} \partial x_{j}}\left(\phi_{2} \circ \phi_{1}^{-1}\right)=0,
$$

where

$$
g_{i j}=\delta_{i j}+\left[\frac{\partial}{\partial x_{i}}\left(\left(\phi_{2}+w\right) \circ \phi_{1}^{-1}\right)\right] \cdot\left[\frac{\partial}{\partial x_{j}}\left(\left(\phi_{2}+w\right) \circ \phi_{1}^{-1}\right)\right],
$$

and $G^{i j}$ is the corresponding matrix of cofactors. Hence on $\Omega_{2}, w$ satisfies a similar system of the form

$$
\sum_{i, j=1}^{k+1} H^{i j} w_{i j}+h=0
$$

It follows from (1) that the $H^{i j}$ are uniformly bounded in $C^{0 . \alpha}$ norm, that $h$ is uniformly bounded in $C^{0, \alpha}$ norm, and that the system is uniformly elliptic. Along $\Omega_{2} \cap \partial \Omega, w=0$. In $\Omega_{2},\|w\|_{C^{1}} \rightarrow 0$. Therefore by Schauder estimates [17, Chapter 3, 1.12 and 1.13], in $\Omega_{3},\|w\|_{C^{2 . \alpha}} \rightarrow 0$. By Lemma 3.2, $\left\|g \mid M \cap C_{4}\right\|_{C^{2, \alpha}} \rightarrow 0$. Since finitely many $C_{4}$ 's cover $M,\|g\|_{C^{2, \alpha}} \rightarrow 0$. Consequently, taking $f=\phi(\gamma)+g$,

$$
T=f_{\#} S \text { and }\left\|f-\iota_{M}\right\|_{C^{2 . \alpha}} \rightarrow 0 .
$$

Finally, since $M$ has no nontangential Jacobi field which vanishes at the boundary, one can apply White's theorem (cf. Remark 3.1 ) to conclude that $T$ is unique. 
3.3. Corollary. A curve in $\mathbf{R}^{n}$ sufficiently $C^{2, \alpha}$ close to a circle bounds a unique area-minimizing flat chain modulo two, which is an embedded disc.

3.4. COROLlary. A regular point in a minimal surface has a neighborhood which is area-minimizing as a flat chain modulo two.

REMARK. Corollary 3.4 improves the older theorem that a regular point in a minimal surface has a neighborhood which is area-minimizing as an integral current (cf. [27]).

Results such as Theorem 4 have been known to workers in the field and are implicit in the literature.

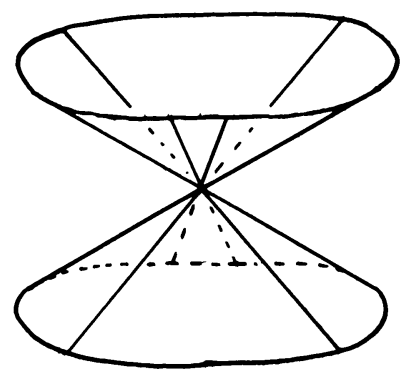

FIGURE 4. The area-minimizing cone over $\mathbf{S}^{3} \times \mathbf{S}^{3}$ in $\mathbf{R}^{8}$, schematic

4. THEOREM. Let $S$ be an ( $n-1)$-dimensional area-minimizing integral current in $\mathbf{R}^{n}$ bounded by a compact, connected submanifold $B$ of the unit sphere. Then $S^{2}$ is area-minimizing in the class of flat chains modulo two.

REMARK. Every flat chain modulo two $Y$ of finite mass has a representative modulo two. The following lemma of B. White gives a simultaneous representation for $Y$ and $\partial Y$ when $Y$ is $n$-dimensional. It says that every compact hypersurface without boundary is orientable.

4.1. LemMa [25, Corollary 2.3]. Let $Y$ be an n-dimensional flat chain modulo two in $\mathbf{R}^{n}$, such that $Y$ and $\partial Y$ have finite mass. Then there is an intgral current $S$ such that $S$ is a representative modulo two for $Y$ and $\partial S$ is a representative modulo two for $\partial Y$.

RemarK. The following lemma asserts that two disjoint hypersurfaces with a common boundary are both orientable. It implies, for example, that any surface with the same boundary as a Möbius strip in $\mathbf{R}^{3}$ must intersect the interior of the Möbius strip.

4.2. Lemma. Let $X_{1}, X_{2}$ be (n-1)-dimensional flat chains modulo two in $\mathbf{R}^{n}$ with $\partial X_{1}=\partial X_{2}$. Suppose that $\mathrm{spt}^{2} X_{1} \cap \mathrm{spt}^{2} X_{2}=\mathrm{spt}^{2} \partial X_{1}$ and $\mathscr{H}^{n-1}\left(\mathrm{spt}^{2} \partial X_{1}\right)=0$. Then $X_{1}$ has a representative modulo two $R$ with spt $\partial R=\operatorname{spt}^{2} \partial X_{1}$.

Proof. Since $\partial X_{1}=\partial X_{2}, X_{1}-X_{2}=\partial Y$, for some flat chain mod 2 of finite mass $Y$. By Lemma 4.1, there is an integral current $S$ such that $\partial S$ is a representative 
$\bmod 2$ for $\partial Y=X_{1}-X_{2}$. Put $R=\partial S\left\llcorner\left(\mathbf{R}^{n}-\operatorname{spt}^{2} X_{2}\right)\right.$. Then $R$ is a representative $\bmod 2$ for $X_{1}$ and spt $\partial R \subset \operatorname{spt}^{2} \partial X_{1}$. Hence spt $\partial R=\operatorname{spt}^{2} \partial X_{1}$.

ProOF OF THEOREM 4. Let $X_{1}$ be any area-minimizing flat chain mod 2 bounded by $B$. We will show that $\mathbf{M}(S) \leqslant \mathbf{M}^{2}\left(X_{1}\right)$. Let $X_{2}$ be a flat chain mod 2 in the unit sphere bounded by $B$. Since $X_{1}$ is area-minimizing, spt $^{2} X_{1}$ intersects the sphere only in $B$; hence,

$$
\mathrm{spt}^{2} X_{1} \cap \mathrm{spt}^{2} X_{2}=B .
$$

By Lemma 4.2, $X_{1}$ has a representative $\bmod 2 R$ with spt $\partial R=\operatorname{spt}^{2} \partial X_{1}=B$. Since $B$ is connected, it follows by $[7,4.1 .31(2)$ and 4.2.27] that $\partial R=k B$ for some nonzero integer $k$. Next it follows from $[\mathbf{8}, 5.10]$ that there is a rectifiable current $T$ with $\partial T=B$ and $\mathbf{M}(T)=|1 / k| \mathbf{M}(R)=|1 / k| M^{2}\left(X_{1}\right)$. Finally, by the minimizing property of $S, \mathbf{M}(S) \leqslant \mathbf{M}(T) \leqslant \mathbf{M}^{2}\left(X_{1}\right)$.

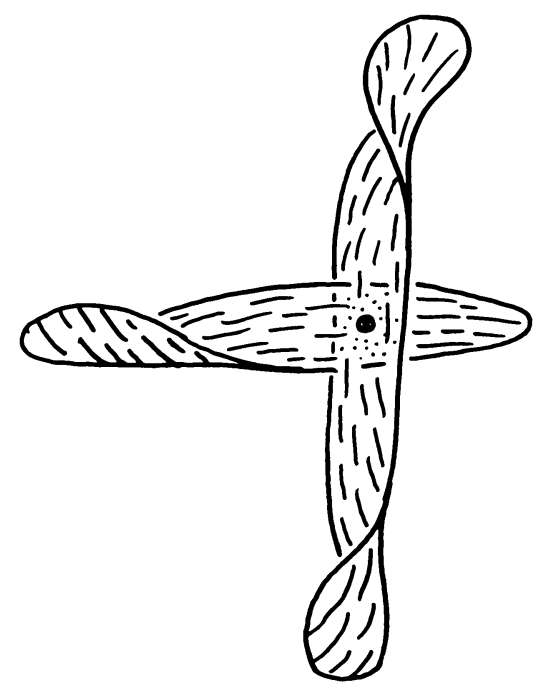

FIGURE 5. The union of two orthogonal area-minimizing surfaces

5. Theorem. For integers $2 \leqslant k \leqslant n$ let $S_{1}, S_{2}$ be $k$-dimensional area-minimizing flat chains modulo two. Suppose that their supports, $\mathrm{spt}^{2} S_{1}$ and $\mathrm{spt}^{2} S_{2}$, lie in subspaces $P_{1}$ and $P_{2}$ of $\mathbf{R}^{n}$ with

$$
\operatorname{dim}\left(P_{1} \cap P_{2}^{\perp}\right) \geqslant \operatorname{dim} P_{1}-k+2 .
$$

Then $S=S_{1}+S_{2}$ is area-minimizing. Suppose, further, that

$$
\operatorname{dim}\left(P_{1} \cap P_{2}\right)<k-2
$$

and $S^{\prime}$ is an area-minimizing flat chain modulo two with $\partial S^{\prime}=\partial S$. Then $S^{\prime}=S_{1}^{\prime}+S_{2}^{\prime}$ with $\mathrm{spt}^{2} S_{1}^{\prime} \subset P_{1}, \mathrm{spt}^{2} S_{2}^{\prime} \subset P_{2}, \partial S_{1}^{\prime}=\partial S_{1}, \partial S_{2}^{\prime}=\partial S_{2}$.

REMARK. The theorem, proof and Corollary 5.4 hold for rectifiable currents as well. In the special case when $P_{1} \perp P_{2}$, it was proved that $S$ is area-minimizing by $\mathrm{H}$. Federer and W. Fleming in 1960 ([11, 9.15], cf. also [13, §2, Example]). The general case is new. 
REMARK. The following lemma also plays a central role in the general study of calibrations. See [21, Lemma 4; 5; 20 and 16].

5.1. Lemma. Consider $\mathbf{R}^{2 m} \cong \mathbf{C}^{m}$ with orthonormal basis $e_{1}, i e_{1}, \ldots, e_{m}$, ie $e_{m}$. Let

$$
\phi \in \bigotimes_{j=1}^{m}\left(\wedge^{1} \operatorname{span}\left\{e_{j}, i e_{j}\right\}\right) \subset \wedge^{m} \mathbf{R}^{2 m} .
$$

Then on the space of unit simple m-vectors, $\phi$ has a maximum point of the form

$$
\exp \left(i \alpha_{1}\right) e_{1} \wedge \exp \left(i \alpha_{2}\right) e_{2} \wedge \cdots \wedge \exp \left(i \alpha_{m}\right) e_{m} .
$$

(5) Furthermore, for any $j$, if there is an $\alpha_{j}^{0}$ such that $\phi$ has no maximum of form (4) with $\alpha_{j}=\alpha_{j}^{0}$, then every maximum point $\xi$ has a factor of the form $\exp \left(i \alpha_{j}\right) e_{j}$.

(6) In particular, if $\phi$ has only finitely many maxima of form (4), then all of the maxima of $\phi$ are of form (4).

Proof. Let $M=\|\phi\| \equiv \max \{\phi(\xi)$ : $\xi$ a unit simple $m$-vector $\}$. It suffices to prove (5), since the other statements are immediate consequences of (5). So assume that there is a $0 \leqslant \alpha_{j}^{0}<2 \pi$ such that $\phi$ has no maximum of form (4) with $\alpha_{j}=\alpha_{j}^{0}$. For convenience we assume $j=1$. It is not hard to show (cf. [15, Lemma II.7.5]) that there are orthonormal vectors $f_{1}, f_{2}, g_{1}, \ldots, g_{m}$ with $\operatorname{span}\left\{f_{1}, f_{2}\right\}=\operatorname{span}\left\{e_{1}, i e_{1}\right\}$ and angles $\theta_{1}, \theta_{2} \in[0, \pi / 2)$ such that $\xi$ takes the form

$$
\xi=\left(\cos \theta_{1} f_{1}+\sin \theta_{1} g_{1}\right) \wedge\left(\cos \theta_{2} f_{2}+\sin \theta_{2} g_{2}\right) \wedge g_{3} \wedge \cdots \wedge g_{m} .
$$

By hypothesis (3),

$$
\phi(\xi)=a \cos \theta_{1} \sin \theta_{2}+b \sin \theta_{1} \cos \theta_{2}
$$

(where $a=\left\langle f_{1} \wedge g_{2} \wedge \cdots \wedge g_{m}, \phi\right\rangle, b=\left\langle g_{1} \wedge f_{2} \wedge \cdots \wedge g_{m}, \phi\right\rangle$ )

$$
\leqslant \sqrt{a^{2} \cos ^{2} \theta_{1}+b^{2} \sin ^{2} \theta_{1}} \leqslant \max \{|a|,|b|\} \leqslant M .
$$

Hence, equality holds. Unless $a=b=M,\left\{\theta_{1}, \theta_{2}\right\}=\{0, \pi / 2\}$ and $\xi$ has as a factor $f_{1}$ or $f_{2}$, as desired. If $a=b=M, f_{1} \wedge g_{2} \wedge \cdots \wedge g_{m}$ is a maximum point of $\phi$, and hence

$$
\begin{aligned}
0 & =\left.\frac{d}{d t}\left\langle f_{1} \wedge\left(\cos t g_{2}+\sin t f_{2}\right) \wedge \cdots \wedge g_{m}, \phi\right\rangle\right|_{t=0} \\
& =\left\langle f_{1} \wedge f_{2} \wedge \cdots \wedge g_{m}, \phi\right\rangle .
\end{aligned}
$$

Similarly, $0=\left\langle g_{1} \wedge g_{2} \wedge \cdots \wedge g_{m}, \phi\right\rangle$. Therefore, for any $\theta$,

$$
\begin{gathered}
\left\langle\left(\cos \theta f_{1}+\sin \theta f_{2}\right) \wedge\left(-\sin \theta g_{1}+\cos \theta g_{2}\right) \wedge g_{3} \wedge \cdots \wedge g_{m}, \phi\right\rangle \\
=M \cos ^{2} \theta+M \sin ^{2} \theta+0+0=M .
\end{gathered}
$$

Choosing $\theta$ so that $\exp \left(i \alpha_{1}^{0}\right) e_{1}=\cos \theta f_{1}+\sin \theta f_{2}$ shows that $\left.\| \exp \left(i \alpha_{1}^{0}\right) e_{1}\right\lrcorner \phi \|=M$. But by induction $\left.\exp \left(i \alpha_{1}^{0}\right) e_{1}\right\lrcorner \phi$ has a maximum of the form $\exp \left(i \alpha_{2}\right) e_{2} \wedge \cdots \wedge$ $\exp \left(i \alpha_{m}\right) e_{m}$. Therefore $\phi$ has a maximum point of the form

$$
\exp \left(i \alpha_{1}^{0}\right) e_{1} \wedge \exp \left(i \alpha_{2}\right) e_{2} \wedge \cdots \wedge \exp \left(i \alpha_{m}\right) e_{m},
$$

and the hypothesis of (5) fails to hold. 
5.2. Lemma. Let $P_{1}, P_{2}$ be subspaces of $\mathbf{R}^{n}$ with

$$
\operatorname{dim}\left(P_{1} \cap P_{2}^{\perp}\right) \geqslant \operatorname{dim} P_{1}-k+2 .
$$

Let $\zeta$ be a unit simple vector in $\Lambda_{k} \mathbf{R}^{n}$. Then the projections of $\zeta$ satisfy $\left|P_{1} \zeta\right|+\left|P_{2} \zeta\right| \leqslant 1$. If furthermore, $\operatorname{dim}\left(P_{1} \cap P_{2}\right)<k-2$, then $\left|P_{1} \zeta\right|+\left|P_{2} \zeta\right|=1$ if and only if $\zeta$ lies in $P_{1}$ or in $P_{2}$.

Remark. The following converse holds. Suppose $P_{1}, P_{2}$ are subspaces of $\mathbf{R}^{n}$ of dimension at least $k$, such that for all unit simple $\zeta \in \Lambda_{k} \mathbf{R}^{n}$,

$$
\left|P_{1} \zeta\right|+\left|P_{2} \zeta\right| \leqslant 1
$$

Then $\operatorname{dim}\left(P_{1} \cap P_{2}^{\perp}\right) \geqslant \operatorname{dim} P_{1}-k+2$. Suppose, furthermore, that equality in (8) holds only if $\zeta$ lies in $P_{1}$ or in $P_{2}$. Then $\operatorname{dim}\left(P_{1} \cap P_{2}\right)<k-2$.

Proof of Lemma 5.2. We may assume that $P_{1} \zeta$ and $P_{2} \zeta$ are nonzero, and for convenience that $n \geqslant 2 k$. Let $u_{1}, u_{2}$ be unit $k$-vectors such that $P_{j} \zeta$ is a positive multiple of $u_{j}$. There are orthonormal vectors

$$
e_{1}, \ldots, e_{k}, i e_{1}, \ldots, i e_{k}
$$

such that

$$
u_{1}=e_{1} \wedge \cdots \wedge e_{k}, \quad u_{2}= \pm \exp \left(i \theta_{1}\right) e_{1} \wedge \cdots \wedge \exp \left(i \theta_{k}\right) e_{k}
$$

with $0 \leqslant \theta_{1} \leqslant \cdots \leqslant \theta_{k} \leqslant \pi / 2$ (cf. [15, II.7.5]). By (7), $\theta_{k-1}=\theta_{k}=\pi / 2$. By replacing $i e_{k}$ by $-i e_{k}$ if necessary, we may assume the ambiguous sign on $u_{2}$ is positive. For any vector $x$, let $x^{*}$ denote the dual covector, and put $\phi=u_{1}^{*}+u_{2}^{*}$. By Lemma 5.1, on the space of unit simple $k$-vectors, $\phi$ has a maximum point $\xi$ of the form

$$
\xi=\exp \left(i \alpha_{1}\right) e_{1} \wedge \cdots \wedge \exp \left(i \alpha_{k}\right) e_{k}
$$

We compute that

$$
\begin{aligned}
\phi(\xi) & =\cos \alpha_{1} \cdots \cos \alpha_{k}+\cos \left(\theta_{1}-\alpha_{1}\right) \cdots \cos \left(\theta_{k}-\alpha_{k}\right) \\
& \leqslant\left|\cos \alpha_{k-1} \cos \alpha_{k}\right|+\left|\cos \left(\theta_{k-1}-\alpha_{k-1}\right) \cos \left(\theta_{k}-\alpha_{k}\right)\right| \\
& =\left|\cos \alpha_{k-1} \cos \alpha_{k}\right|+\left|\sin \alpha_{k-1} \sin \alpha_{k}\right| \\
& \leqslant\left(\cos ^{2} \alpha_{k-1}+\sin ^{2} \alpha_{k-1}\right)^{1 / 2}\left(\cos ^{2} \alpha_{k}+\sin ^{2} \alpha_{k}\right)^{1 / 2}=1
\end{aligned}
$$

Therefore,

$$
\left|P_{1} \zeta\right|+\left|P_{2} \zeta\right|=\phi(\zeta) \leqslant \phi(\xi) \leqslant 1 .
$$

If, furthermore, $\operatorname{dim}\left(P_{1} \cap P_{2}\right)<k-2$, then $\theta_{k-2}>0$. We suppose that $\left|P_{1} \zeta\right|+$ $\left|P_{2} \zeta\right|=\phi(\zeta)=1$ and show that $\zeta=u_{1}$ or $\zeta=u_{2}$, which will prove that $\zeta$ lies in $P_{1}$ or in $P_{2}$. By 5.1(6), it suffices to show that $u_{1}$ and $u_{2}$ are the only maxima of $\phi$ of the form (4). But for any such maximum $\xi$, equality must hold in (10). Since $\theta_{k-2}>0$, either $\alpha_{1}=\cdots=\alpha_{k}=0$ or $\left(\theta_{1}-\alpha_{1}\right)=\cdots=\left(\theta_{k}-\alpha_{k}\right)=0$. Hence $\xi=u_{1}$ or $\xi=u_{2}$.

5.3. Corollary to Lemma. Let $P_{1}, P_{2}$ be subspaces of $\mathbf{R}^{n}$ with $\operatorname{dim}\left(P_{1} \cap P_{2}^{\perp}\right) \geqslant$ $\operatorname{dim} P_{1}-k+2$. Let $T$ be any flat chain modulo two of finite mass. Then

$$
\mathbf{M}^{2}(T) \geqslant \mathbf{M}^{2}\left(P_{1 \#} T\right)+\mathbf{M}^{2}\left(P_{2 \#} T\right) .
$$


Furthermore, if $\operatorname{dim}\left(P_{1} \cap P_{2}\right)<k-2$ and equality holds in (11), then for $\|T\|^{2}$-almost all $x \in \mathbf{R}^{n}, \vec{T}$ lies in $P_{1}$ or in $P_{2}$.

Proof of Theorem 5. Let $T \in \mathscr{R}_{k}^{2} \mathbf{R}^{n}$ with $\partial T=\partial S$. Then by Corollary 5.3,

$$
\mathbf{M}^{2}(T) \geqslant \mathbf{M}^{2}\left(P_{1 \#} T\right)+\mathbf{M}^{2}\left(P_{2 \#} T\right) \geqslant \mathbf{M}^{2}\left(S_{1}\right)+\mathbf{M}^{2}\left(S_{2}\right)=\mathbf{M}^{2}(S)
$$

because $S_{1}$ and $S_{2}$ are area-minimizing and $\partial P_{j \#} T=\partial P_{j \#} S=\partial S_{j}$. Hence $S$ is area-minimizing.

Furthermore, suppose that $\operatorname{dim}\left(P_{1} \cap P_{2}\right)<k-2$ and $\mathbf{M}^{2}(T)=\mathbf{M}^{2}(S)$. By Corollary 5.3, $T$ has a decomposition $T=T_{1}+T_{2}$ in $\mathscr{R}_{k}^{2} \mathbf{R}^{n}$ such that for $\left\|T_{j}\right\|$-almost all $x \in \mathbf{R}^{n}, \vec{T}_{j}$ lies in $P_{j}$ and $\mathbf{M}^{2}(T)=\mathbf{M}^{2}\left(T_{1}\right)+\mathbf{M}^{2}\left(T_{2}\right)$. Let $X=\partial T_{1}-\partial S_{1}=\partial S_{2}$ $-\partial T_{2} \in \mathscr{F}_{k-1}^{2} \mathbf{R}^{n}$. Since $P_{1 \#} X=P_{1 \#}\left(\partial S_{2}-\partial T_{2}\right)=\partial\left(P_{1 \#} S_{2}-P_{1 \#} T_{2}\right)=0, \mathrm{spt}^{2} X$ $\subset P_{2}$. Similarly $\mathrm{spt}^{2} X \subset P_{1}$, so that $\operatorname{spt}^{2} X \subset P_{1} \cap P_{2}$. Now $X=\partial Y$ for some $Y \in \mathscr{R}_{k}^{2} \mathbf{R}^{n}$ with $\operatorname{spt}^{2} Y \subset P_{1} \cap P_{2}$. But since $\mathscr{H}^{k}\left(P_{1} \cap P_{2}\right)=0, Y=0$ (cf. [7, p. 432]). Therefore $X=0, \partial T_{1}=\partial S_{1}$ and $\partial T_{2}=\partial S_{2}$. Since

$$
\mathbf{M}^{2}\left(T_{1}\right)+\mathbf{M}^{2}\left(T_{2}\right)=\mathbf{M}^{2}(T)=\mathbf{M}^{2}(S)=\mathbf{M}^{2}\left(S_{1}\right)+\mathbf{M}^{2}\left(S_{2}\right),
$$

$T_{1}$ and $T_{2}$ are area-minimizing. Hence spt $T_{1} \subset P_{1}$ and spt $T_{2} \subset P_{2}$.

5.4. Corollary to Theorem. Let $R_{1}, R_{2}$ be l-dimensional area-minimizing flat chains modulo two in $\mathbf{R}^{n}$, regular on the interior; i.e., $M_{i}=\mathrm{spt}^{2} R_{i}-\mathrm{spt}^{2} \partial R_{i}$ is a smooth manifold. Then $M_{1} \cap M_{2}$ is the interior singular set of some area-minimizing flat chain modulo two. More precisely, with $m=n-l+2$, there is an area-minimizing flat chain modulo two $T \in \mathscr{R}_{l+m} \mathbf{R}^{n+2 m}$ such that the set of points where $\operatorname{spt}^{2} T-$ spt $^{2} \partial T$ fails to be a smooth manifold is $\left(M_{1} \cap M_{2}\right) \times\{0\} \subset \mathbf{R}^{n} \times \mathbf{R}^{2 m}$. In particular, taking $R_{2}=R_{1}$, we see that the interior of any regular area-minimizing flat chain modulo two is the interior singular set of some other area-minimizing flat chain modulo two.

REMARKS. From two-dimensional area-minimizing flat chains modulo two in $\mathbf{R}^{3}$, the above corollary constructs five-dimensional area-minimizing flat chains modulo two in $\mathbf{R}^{9}$ with interior singular sets consisting of a smooth nonlinear curve or of an even number of nonlinear curves meeting at a point. These seem to be the first examples of nonlinear singular sets in area-minimizing flat chains modulo two. The corollary also holds for rectifiable currents and provides the first examples of nonlinear curves as singular sets in area-minimizing rectifiable currents.

PRoof. Let $k=l+m$ and define flat chains $\bmod 2, S_{1}, S_{2} \in \mathscr{R}_{k}^{2}\left(\mathbf{R}^{n} \times \mathbf{R}^{m} \times \mathbf{R}^{m}\right)$, as the following Cartesian products of $R_{1}, R_{2}$ and $m$-cubes:

$$
S_{1}=R_{1} \times[-1,1]^{m} \times\{0\}, \quad S_{2}=R_{2} \times\{0\} \times[-1,1]^{m} .
$$

Let $T=S_{1}+S_{2} . S_{1}$ and $S_{2}$ are area-minimizing flat chains mod 2 (cf. Remark on Theorem 6), supported in $P_{1}=\mathbf{R}^{n} \times \mathbf{R}^{m} \times\{0\}$ and $P_{2}=\mathbf{R}^{n} \times\{0\} \times \mathbf{R}^{m}$. Since

$$
\operatorname{dim}\left(P_{1} \cap P_{2}^{\perp}\right)=m=n-l+2=m+n-k+2=\operatorname{dim} P_{1}-k+2,
$$

Theorem 5 implies that $T=S_{1}+S_{2}$ is area-minimizing. It is easy to check that $T$ has the desired singular set.

REMARK ON THEOREM 6. It is well known that if $S$ is an area-minimizing flat chain modulo two, then the Cartesian product of $S$ with any interval is area-minimizing. 
The following theorem, under merely a stability hypothesis on $S$, concludes that the Cartesian product of $S$ with a sufficiently short interval is area-minimizing.

6. THEOREM. Suppose $S$ is a $k$-dimensional flat chain modulo two in $\mathbf{R}^{n}$, $\partial S$ has finite mass, and $S$ satisfies the following stability condition: there exists $\varepsilon>0$ such that if $T$ is a flat chain modulo two, $\partial T=\partial S$, and $\mathscr{F}^{2}(T-S)<\varepsilon$, then $\mathbf{M}^{2}(T) \geqslant \mathbf{M}^{2}(S)$.

Then there is $a \delta>0$ such that the Cartesian product of $S$ with an interval of length $\delta$ is area-minimizing in $\mathbf{R}^{n+1}$.

Proof. Take any $\delta<\varepsilon / \mathbf{N}^{2}(S)\left(\mathbf{N}^{2}(S)=\mathbf{M}^{2}(S)+\mathbf{M}^{2}(\partial S)\right)$. Assume that $S \times$ $[0, \delta]^{2}$ is not area-minimizing. Then there is a $Q \in \mathbf{I}_{k+2}^{2}\left(\mathbf{R}^{n+1}\right)$ with

$$
\partial Q=\partial\left(S \times[0, \delta]^{2}\right) \text { and } \mathbf{M}^{2}(Q)<\mathbf{M}^{2}\left(S \times[0, \delta]^{2}\right) .
$$

We may assume $\operatorname{spt}^{2}\left(Q-S \times[0, \delta]^{2}\right) \subset \mathbf{R}^{n} \times[0, \delta]$. In fact, by increasing $\delta$ slightly, we may assume $\operatorname{spt}^{2}\left(Q-S \times[0, \delta]^{2}\right) \subset \mathbf{R}^{n} \times(0, \delta]$. For $r \in[0, \delta]$ let

$$
Q_{r}=\left\langle Q, x_{n+1}, r+\right\rangle
$$

be the slice of $Q$ by the hyperplane $x_{n+1}=r$ (cf. [7, p. 429]). Since

$$
\int_{r=0}^{\delta} \mathbf{M}^{2}\left(Q_{r}\right) d r \leqslant \mathbf{M}^{2}(Q)<\mathbf{M}^{2}\left(S \times[0, \delta]^{2}\right)=\int_{r=0}^{\delta} \mathbf{M}^{2}(S) d r,
$$

we can choose $r \in(0, \delta)$ such that $\mathbf{M}^{2}\left(Q_{r}\right)<\mathbf{M}^{2}(S)$. Since

$$
Q_{r}-Q_{0}=\partial\left[Q\left\llcorner\left\{0<x_{n+1} \leqslant r\right\}\right]-(\partial Q)\left\llcorner\left\{0<x_{n+1} \leqslant r\right\},\right.\right.
$$

we have

$$
\begin{aligned}
\mathscr{F}^{2}\left(Q_{r}-Q_{0}\right) & \leqslant \mathbf{M}^{2}(Q)+\mathbf{M}^{2}(\partial Q)\left\llcorner\left\{0<x_{n+1}<\delta\right\}\right. \\
& <\mathbf{M}^{2}\left(S \times\lceil 0, \delta\rceil^{2}\right)+\mathbf{M}^{2}\left((\partial S) \times\lceil 0, \delta]^{2}\right) \\
& \leqslant \delta \mathbf{N}^{2}(S) .
\end{aligned}
$$

Now let $p$ denote orthogonal projection of $\mathbf{R}^{n} \times \mathbf{R}$ into $\mathbf{R}^{n}$. Since

$$
\begin{gathered}
\operatorname{spt}\left(Q-S \times[0, \delta]^{2}\right) \subset \mathbf{R}^{n} \times(0, \delta], \\
p_{\#} Q_{0}=p_{\#}\left(\left\langle S \times[0, \delta]^{2}, x_{n+1}, 0\right\rangle\right)=S .
\end{gathered}
$$

Put $T=p_{\#} Q_{r} \in \mathscr{R}_{k}^{2} \mathbf{R}^{n}$. One computes that

$$
\begin{aligned}
\partial T & =p_{\#}\left\langle\partial Q, x_{n+1}, r+\right\rangle=p_{\#}\left\langle\partial\left(S \times[0, \delta]^{2}\right), x_{n+1}, r+\right\rangle \\
& =p_{\#}\left(\partial S \times \delta_{r}\right)=\partial S, \\
\mathscr{F}^{2}(T & -S)=\mathscr{F}^{2}\left(p_{\#}\left(Q_{r}-Q_{0}\right)\right) \leqslant \mathscr{F}^{2}\left(Q_{r}-Q_{0}\right)<\delta \mathbf{N}^{2}(S)<\varepsilon .
\end{aligned}
$$

But $\mathbf{M}^{2}(T) \leqslant \mathbf{M}^{2}\left(Q_{r}\right)<\mathbf{M}^{2}(S)$, a contradiction of the stability condition on $S$.

6.1. Question. Is the Cartesian product of two area-minimizing flat chains modulo two area-minimizing?

(Although, in general, the Cartesian product of rectifiable currents need not be rectifiable, the almost everywhere regularity of area-minimizing flat chains modulo two insures that their product is a flat chain modulo two of finite mass.) The question is open also for normal currents (cf. [20]) but settled negatively for integral currents (cf. $[19,1.2])$. 


\section{REFERENCES}

1. William K. Allard, On the first variation of a varifold, Ann. of Math. (2) 95 (1972), 417-491.

2. __ On the first variation of a varifold: Boundary behavior. Ann. of Math. (2) 101 (1975), $418-446$

3. F. J. Almgren, Jr., How to connect minimal surfaces by bridges, in preparation. (Cf. Abstracts Amer. Math. Soc. 3 (1980), 775 B7, p. 225.)

4. _ $\mathbf{Q}$ valued functions minimizing Dirichlet's integral and the regularity of area minimizing rectifiable currents up to codimension two (preprint).

5. J. Dadok and R. Harvey, Calibrations on $\mathbf{R}^{6}$, Duke Math. J. (to appear).

6. Ennio DeGiorgi, Sulla differenziabilità e l'analiticità delle estremali degli integrali multipli regolari, Mm. Accad. Sci. Torino Cl. Sci. Fis. Mat. Natur. (3) 3 (1957), 25-43.

7. Herbert Federer, Geometric measure theorey, Springer-Verlag, Heidelberg and New York, 1969.

8. $351-407$.

9. minimizing flat chains modulo two with arbitrary codimension, Bull. Amer. Math. Soc. 76 (1970), 767-771.

10. __ Some theorems on integral currents, Trans. Amer. Math. Soc. 117 (1965), 43-67.

11. Herbert Federer and Wendell H. Fleming, Normal and integral currents, Ann. of Math. (2) 72 (1960), 458-520.

12. Wendell H. Fleming, Flat chains over a coefficient group, Trans. Amer. Math. Soc. 121 (1966), $160-186$.

13. __ On the oriented Plateau problem, Rend. Circ. Mat. Palermo (2) 11 (1962), 1-22.

14. Robert M. Hardt, On boundary regularity for integral currents or flat chains modulo two minimizing the integral of an elliptic integrand, Comm. Partial Differential Equations 2 (1977), 1163-1232.

15. Reese Harvey and H. Blaine Lawson, Jr., Calibrated geometries, Acta Math. 148 (1982), 47-157.

16. Reese Harvey and Frank Morgan, The comass ball in $\wedge^{3} \mathbf{R}^{6}$, in preparation.

17. Olga A. Ladyzhenskaya and Nina N. Ural'tseva, Linear and quasilinear elliptic equations, Academic Press, New York, 1968.

18. H. Blaine Lawson, Jr., The equivariant Plateau problem and interior regularity, Trans. Amer. Math. Soc. 173 (1972), 231-249.

19. Frank Morgan, Area-minimizing currents bounded by higher multiples of curves, Rend. Circ. Mat. Palerno 33 (1984).

20. __ The exterior algebra $\wedge^{k} \mathbf{R}^{n}$ and area minimization, Linear Algebra Appl. (to appear).

21. On the singular structure of three-dimensional, area-minimizing surfaces, Trans. Amer. Math. Soc. 276 (1983), 137-143.

22. _ A smooth curve in $\mathbf{R}^{4}$ bounding a continuum of area minimizing surfaces, Duke Math. J. 43 (1976), 867-870.

23. Robert Osserman, Minimal varieties, Bull. Amer. Math. Soc. 75 (1969), 1092-1120.

24. Brian White, The space of $m$-dimensional surfaces that are stationary for a parametric elliptic integrand (preprint).

25. The structure of minimizing hypersurfaces mod 4, Invent. Math. 53 (1979), 45-58.

26. W. P. Ziemer, Integral currents mod 2, Trans. Amer. Math. Soc. 105 (1962), 496-524.

27. Herbert Federer, A minimizing property of extremal submanifolds, Arch. Rational Mech. Anal. 59 (1975), 207-217.

Department of Mathematics, Massachusetts Institute of Technology, Cambridge, MasSACHUSETTS 02139 